\title{
MicroRNA-133b/EGFR axis regulates esophageal squamous cell carcinoma metastases by suppressing anoikis resistance and anchorage-independent growth
}

Jin-Feng Zhu' ${ }^{2}$, Yi Liư ${ }^{3}$, He Huang ${ }^{4,5}$, Li Shan ${ }^{1}$, Zhi-Gang Han ${ }^{1}$, Jun-Yuan Liu' ${ }^{1}$, Ying-Long Li ${ }^{1}$, Xiang Dong ${ }^{6}$ and Wei Zeng ${ }^{1,7^{*}}$

\begin{abstract}
Background: Anoikis resistance has been demonstrated to facilitate distant metastases of cancers. MicroRNA-133b (miR-133b) is found to be down-regulated in various tumors, including esophageal squamous cell carcinoma (ESCC), and closely correlates with the malignant phenotype of ESCC. This study aimed to evaluate the roles of miR-133b in metastases of ESCC via regulating anoikis.

Methods: The expression of miR-133b and related molecules were detected in ESCC tissues and cells. The target relationship between miR-133b and epidermal growth factor receptor (EGFR) was verified by dual luciferase reporter assay. Cell proliferation was detected by 3-(4,5-dimethylthiazol-2-yl)-2,5-diphenyltetrazolium bromide assay. Anoikis and anchorage-independent growth were assessed by anoikis assay and soft agar assay. Migration and invasion were evaluated by scratch and transwell assays. The expressions of related molecules were detected by reverse transcription-quantitative polymerase chain reaction and western blotting. The in vivo results were determined by tumor xenografts in nude mice.

Results: MiR-133b level was decreased in ESCC tissues and cells, which negatively correlated with EGFR, integrin $\beta 4$ (ITGB4), and phosphorylated focal adhesion kinase levels. Moreover, miR-133b down-regulated EGFR expression in ESCC cells. Overexpression of miR-133b inhibited the anoikis resistance, migration, invasion and epithelial-mesenchymal transition of ESCC cells via targeting EGFR. Finally, miR-133b overexpression suppressed tumor growth and lung metastases of ESCC in vivo. ITGB4/FAK/growth factor receptor-bound protein 2 (Grb2), protein kinase B (AKT), and extracellular signal-regulated kinase (ERK) pathways were involved in the regulatory mechanisms of miR-133b/EGFR axis in ESCC metastases in vitro and in vivo.
\end{abstract}

Conclusions: The results suggested that miR-133b/EGFR axis regulated metastases of ESCC by affecting anoikis resistance via ITGB4/FAK/Grb2, AKT, and ERK pathways.

Keywords: Esophageal squamous cell carcinoma, miR-133b, Epidermal growth factor receptor, Metastases, Anoikis, Anchorage-independent growth

\footnotetext{
*Correspondence: zengwei1409@163.com

${ }^{1}$ First Department of Lung Cancer Chemotherapy, Affiliated Cancer Hospital of Xinjiang Medical University, No. 789, East Suzhou Street,

Urumqi 830011, Xinjiang, People's Republic of China

Full list of author information is available at the end of the article
} 


\section{Background}

Esophageal squamous cell carcinoma (ESCC), as the main histological type of esophageal carcinoma, is the fourth most common cancer in China [1, 2]. ESCC has been demonstrated to have rapid progression, strongly potential invasion, high frequent metastasis and poor survival [3]. Currently, surgery, chemotherapy and radiotherapy are the common therapeutic methods for ESCC. Whereas the prognosis of ESCC is still poor and the 5 -year survival rate is only about $15-25 \%[4,5]$. Regional or distant metastasis is a risk factor for the recurrence and poor prognosis of ESCC. Therefore, it is urgent to identify new markers and study the mechanisms for recurrence and metastasis to enhance the survival rate of ESCC patients.

MicroRNAs (miRNAs) are short endogenous non-coding RNAs that repress the expression of their target genes via binding to the $3^{\prime}$-untranslated regions (3'-UTRs). Growing evidences have shown that some miRNAs are aberrantly expressed and play pivotal roles in the regulation of growth and metastasis of ESCC. As one of well documented miRNAs, miR-133b has been confirmed to be a tumor suppressor that inhibits the progression of various cancers [6-8]. In ESCC, miR-133b has been also identified to be down-regulated and involved in the malignant phenotype of ESCC $[9,10]$. However, the regulatory mechanisms of miR-133b in the development and progression of ESCC have not been fully elucidated.

Epidermal growth factor receptor (EGFR) that belongs to the human epidermal growth factor receptor family, acts as a proto-oncogene, and promotes cell growth and metastasis [11]. Previous studies demonstrated that miR-133b could restrain cell invasion and metastasis via targeting EGFR in a variety of cancer cells [12-14], including ovarian cancer, non-small-cell lung cancer, and colorectal cancer. However, whether miR-133b can modulate metastases in ESCC through regulating EGFR expression has not been elucidated.

Frisch and Francis discovered anoikis for the first time in 1994 [15]. Anoikis is a programmed cell death that prevents the growth of cells after they separate from the extracellular matrix (ECM) [16]. However, the disorder of gene expression helped the cancer cells to escape anoikis, which resulted in the survival of cancer cells in lymph, blood and facilitated their regional or distant metastasis $[17,18]$. Thus, inducing anoikis of cancer cells may be an effective treatment for suppressing cancer metastasis. A previous study showed that $N$-acetylglucosaminyltransferase $\mathrm{V}$ gene promoted anoikis resistance during metastasis of cancers [19]. Hu et al. demonstrated that inducing caspase-mediated anoikis inhibited the progression of hepatocellular carcinoma [20]. Anchorageindependent growth is a feature of highly invasive tumor cells, because these cells have a better chance of survival and proliferation in the absence of extracellular matrix, then expand and invade adjacent tissues, and further give rise to metastasis [21]. Anoikis resistance is an important protective process for anchorage-independent growth [22]. It has been shown that promoting anchorage-independent growth contributed to the invasion and progression of lung cancer cells [23]. But so far, the roles of miR-133b/EGFR axis in the regulation of anoikis resistance and anchorage-independent growth of ESCC have not been reported.

It was shown that EGFR cooperated with integrin $\beta 4$ (ITGB4) to promote anchorage-independent growth and metastasis of hepatocellular carcinoma [24]. ITGB4 belongs to the integrin family and has been reported to influence carcinoma progression via modulating anoikis. Focal adhesion kinase (FAK) is a protein tyrosine kinase that mediates cell adhesion, and the activation of FAK can ligate conformation of integrin to promote cell proliferation via downstream phosphatidylinositol 3-kinase (PI3K)/Protein kinase B (AKT) pathway, which finally lead to anoikis resistance [25]. Growth factor receptorbound protein 2 (Grb2) is a member of integrin adhesive and critical for the malignant progression of tumors [26]. Previous study found that Grb2/extracellular signal-regulated kinase (ERK)/caspase-3 signaling pathway was involved in anoikis resistance in breast cancer cells [27]. Moreover, Grb2 was suggested to bind to FAK and regulate the proliferation and invasion of melanoma [28]. AKT and ERK are two important downstream signaling pathways regulated by Grb2 and then facilitate tumor progression $[29,30]$. Therefore, ITGB4/FAK/Grb2, AKT and ERK pathways participate in the regulation of anoikis resistance.

In this study, we investigated the roles of miR-133b/ EGFR axis in the metastases of ESCC through modulating anoikis resistance and anchorage-independent growth via ITGB4/FAK/Grb2, AKT and ERK signaling pathways. The research was performed at the clinical, cell, and animal levels, which provided evidences for miR-133b as a therapeutic target for the treatment of ESCC metastasis.

\section{Methods \\ Patient samples}

A total of 30 pairs of ESCC tissues and corresponding adjacent normal tissues were collected from patients with informed consents in the Affiliated Cancer Hospital of Xinjiang Medical University (Urumchi, Xinjiang, China). The experimental protocol was approved by the research ethics committee of the Affiliated Cancer Hospital of Xinjiang Medical University. All patients were not received chemotherapy or radiotherapy before 
surgery. The characteristics of ESCC patients are shown in Table 1 . The samples were immediately snapped frozen in liquid nitrogen till further analysis.

\section{Cell culture and transfection}

Human ESCC cell lines KYSE30, KYSE150, and ECa109 (Type Culture Collection of the Chinese Academy of Sciences, Shanghai, China) were cultured in Roswell Park Memorial Institute (RPMI) 1640 supplemented with 10\% fetal bovine serum (FBS). Normal human esophageal epithelial cell line Het-1A (Cobioer Biosciences, Nanjing, Jiangsu, China) was maintained in Dulbecco's Modified Eagle Medium (DMEM) with 10\% FBS. All cells were cultured at $37{ }^{\circ} \mathrm{C}$ in a humidified atmosphere containing $5 \%$ $\mathrm{CO}_{2}$.

KYSE150 and ECa109 cells were transfected with miR-133b agomir/antagomir (GenePharma, Shanghai, China), or shEGFR (GenePharma) using Lipofectamine ${ }^{\mathrm{R}}$ RNAiMAX Transfection Reagent (Invitrogen, Carlsbad, CA, USA) according to the instructions.

\section{Dual luciferase reporter assay}

The plasmids containing wild-type (WT) or mutated (MUT) 3'-UTR of EGFR were obtained from GeneCopoeia (Guangzhou, Guangdong, China). HEK-293T cells were seeded into 96-well plates and co-transfected with $100 \mathrm{ng}$ luciferase reporter constructs (EGFR-WT or EGFR-MUT) and $100 \mathrm{nM}$ miR-133b agomir or miR133b negative control using Lipo2000 (Invitrogen). At $48 \mathrm{~h}$ after the transfection, luciferase activity was assessed using a Dual-Luciferase Reporter Assay System

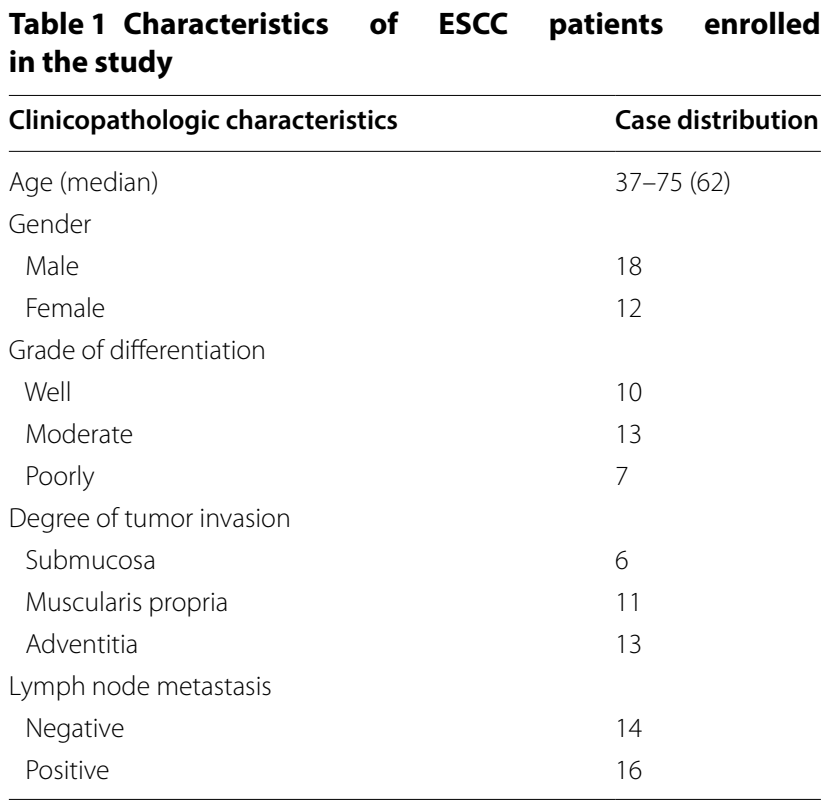

(Promega, Madison, Wisconsin, USA). The dual luciferase reporter assay was performed for three times.

\section{3-(4,5-dimethylthiazol-2-yl)-2,5-diphenyltetrazolium bromide (MTT) assay}

MTT assay was performed to evaluate cell proliferation. Briefly, the transfected cells were seeded into 96-well plates and incubated for $24 \mathrm{~h}$. Then at the indicated time points, $5 \mathrm{mg} / \mathrm{mL}$ MTT (Sigma, Saint Louis, MO, USA) was added into each well. After incubation for $4 \mathrm{~h}$, the supernatant was removed and $200 \mu \mathrm{L}$ of dimethyl sulfoxide (DMSO, Sigma) was added to dissolve the formazan products. The results were read at $490 \mathrm{~nm}$ by a microplate reader (BioTek, Winsky, Vermont, USA).

\section{Anoikis assessment assay}

KYSE150 and ECa109 cells were seeded into poly-Hema (Sigma) pre-coated plates and incubated at $37{ }^{\circ} \mathrm{C}$ for $24 \mathrm{~h}$. Then, the cells were collected, washed with cold phosphate buffer solution (PBS) and subjected to flow cytometry analysis using an Annexin V/FITC Apoptosis Detection Kit (BD Pharmingen $^{\mathrm{TM}}$, Franklin lake, New Jersey, USA) according to the manufacture's instructions.

\section{Soft agar cloning assay for anchorage-independent growth}

About 1500 cells/well were suspended in pre-warmed culture medium containing $0.25 \%$ agarose, and seeded in culture dishes that were pre-coated with $0.5 \%$ agarose in culture medium. Cells were incubated for 2-3 weeks at $37{ }^{\circ} \mathrm{C}$ under a $5 \% \mathrm{CO}_{2}$ atmosphere. The formed colonies were counted and imaged under a light microscope (Olympus, Tokyo, Japan).

\section{Scratch assay}

Cell migration ability was determined by scratch assay. In brief, the transfected cells $\left(0.5 \times 10^{6}\right.$ cells/well $)$ were seeded into 6 -well plates. Then, a scratch was made in the confluent monolayer cells using a $10 \mu \mathrm{L}$ pipette tip. After washing with PBS to remove the cell debris, the images were photographed by a light microscope immediately. Then the cells were maintained in serum-free medium for $24 \mathrm{~h}$ at $37^{\circ} \mathrm{C}$ and photographed. The migration rate of cells was measured by the following formula: $\left(\mathrm{W}_{0 \mathrm{~h}}-\mathrm{W}_{24 \mathrm{~h}}\right) / \mathrm{W}_{0 \mathrm{~h}} \times 100 \%$.

\section{Transwell assay}

Cell invasion ability was assessed by transwell assay. Cells in serum-free medium were seeded in the upper compartments of transwell chambers (Corning, Corning, MA, USA) that were pre-coated with Matrigel. As a chemoattractant, the bottom compartments were added with RPMI1640 containing 20\% FBS. After incubation for 
$16 \mathrm{~h}$ at $37^{\circ} \mathrm{C}$, the non-invaded cells on the upper surface were erased with a cotton swab and the invaded cells on the lower surface were fixed with $4 \%$ paraformaldehyde and stained with Giemsa. The images were photographed in random fields under a light microscope (Olympus, Tokyo, Japan).

\section{Tumor xenografts in nude mice}

Male 7-week-old BALB/c nude mice were purchased from Chinese Academy of Science (Shanghai, China) and maintained under pathogen-free condition. All animal experiments were performed in accordance with the Guide for the Care and Use of Laboratory Animals and approved by the Institutional Ethics committee of the Affiliated Cancer Hospital of Xinjiang Medical University. To evaluate the role of miR-133b in tumor formation, $5 \times 10^{6}$ KYSE150 and ECa109 cells that infected with lentivirus expressing miR-133b agomir or miR-133b NC (GeneChem, Shanghai, China) were injected subcutaneously into the axilla of nude mice. The mice were randomly divided into four groups ( $\mathrm{n}=5$ per group): KYSE150-miR-133b NC, KYSE150-miR-133b agomir, ECa109-miR-133b NC, ECa109-miR-133b agomir. The length and width of the tumors were measured every 5 days and the tumor volume was calculated according to the formula of $0.5 \times$ length $\times$ width $^{2}$. At 30 days after the injection, the mice were sacrificed and the tumors were collected and weighed. To determine lung metastasis, $5 \times 10^{6}$ the above cells were injected into the nude mice via tail vein. Thirty days later, the mice were killed and the lung tissues were collected, and stored in liquid nitrogen for further tests.

\section{Reverse transcription-quantitative polymerase chain reaction (RT-qPCR) assay}

Total RNAs were isolated from ESCC tissues and cells by Trizol (Invitrogen, USA) and reversely transcribed using the PrimerScript RT reagent Kit (TaKaRa, Osaka, Japan). Real-time PCR was performed to assess mRNA and miRNA levels using SYBR Green RT-qPCR SuperMix Kit (Thermo Fisher Scientific, Waltham, Massachusetts, USA) with specific primers (Table 2) on AB7300 thermo-recycler (Applied Biosystems, Foster City, California, USA). Glyceraldehyde-3-phosphate dehydrogenase (GAPDH) and U6 small nuclear RNA (U6 snRNA) were used as internal controls for mRNA and miRNA, respectively. The levels of miR-133b and mRNAs were calculated by the $2^{-\Delta \Delta \mathrm{Ct}}$ method.

\section{Western blotting assay}

Proteins were extracted with radio immunoprecipitation assay (RIPA) (Beyotime, Haimen, Jiangsu, China) containing $1 \%$ phenylmethanesulfonyl fluoride (PMSF)
Table 2 Oligonucleotide primer sets for real-time PCR

\begin{tabular}{lll}
\hline Name & Sequence $\mathbf{( 5}^{\prime} \mathbf{- 3}^{\prime}$ ) & Length \\
\hline miR-133b-F & TTTGGTCCCCTTCAACCAGCTA & 22 \\
miR-133b-R & GTGCAGGGTCCGAGGT & 16 \\
EGFR-F & CACTGCCTCATCTCTCACCATC & 22 \\
EGFR-R & GACTCACCGTAGCTCCAGAC & 20 \\
ITGB4-F & GCGACTACACTATTGGATTTGGC & 23 \\
ITGB4-R & TGTCAGGCTGATGACGTTCTTG & 22 \\
FAK-F & CATCCCTAACCATTGCG & 17 \\
FAK-R & GCCCGTTCACCTTCTTT & 17 \\
Grb2-F & AAGACGGCTTCATTCCCAAG & 20 \\
Grb2-R & CTCTCTCGGATAAGAAAGGC & 20 \\
GAPDH-F & CAGGGCTGCTTTTAACTCTGGT & 22 \\
GAPDH-R & GATTTTGGAGGGATCTCGCT & 20 \\
U6-F & CGCAAGGATGACACGCAAATTC & 22 \\
U6-R & GTGCAGGGTCCGAGGT & 16 \\
\hline
\end{tabular}

from ESCC tissues and cells followed by centrifugation at $14,000 \mathrm{~g}$ at $4{ }^{\circ} \mathrm{C}$ for $10 \mathrm{~min}$. The proteins in supernatant were collected and quantified by a bicinchoninic acid (BCA) Protein Assay kit (Thermo Fisher Scientific). Then $40 \mu \mathrm{g}$ protein samples were subjected to sodium dodecyl sulphate-polyacrylamide gel electrophoresis (SDS-PAGE) and transferred onto polyvinylidene fluoride membranes (Millipore, Massachusetts, USA). Subsequently, the membranes were incubated with $5 \%$ skim milk for $1 \mathrm{~h}$ to block the non-specific binding and probed with primary antibodies against EGFR (1:2000, Abcam, Cambridge, UK), ITGB4 (1:1000, Abcam), p-FAK (1:1000, Abcam), FAK (1:1000, Abcam), Fibronectin (1:1000, Abcam), Vimentin (1:1000, Cell Signaling Technology, Danvers, MA, USA), N-cadherin (1:1000, Cell Signaling Technology), E-cadherin (1:1000, Cell Signaling Technology), matrix metalloproteinase 2 (MMP-2, 1:1000, Proteintech, Rosemont, Illinois, USA), MMP-9 (1:1000, Proteintech), Grb2 (1:1000, Proteintech), p-AKT $\mathrm{Thr}_{308}$ (1:1000, Cell Signaling Technology), p-AKT $\mathrm{Aer}_{\mathrm{S} 43}(1: 2000$, Cell Signaling Technology), AKT (1:1000, Cell Signaling Technology), p-ERK1/2 (1:1000, Abcam), ERK1/2 (1:1000, Abcam), GAPDH (1:5000, Proteintech) at $4{ }^{\circ} \mathrm{C}$ overnight, respectively. Then, the membranes were incubated with horseradish peroxidase-conjugated goat anti-mouse or anti-rabbit (1:5000, Beyotime) secondary antibody for $1 \mathrm{~h}$ at room temperature and visualized using ECL reagent (Millipore).

\section{Statistical analysis}

All experiments were performed at least for three times with one representative experiment shown. Data were expressed as mean \pm standard deviation (SD). Statistical analysis was performed using Student's $t$ test (two tailed) between two groups or one-way analysis of variance 
(ANOVA) followed by Tukey post hoc test for multiple comparison by SPSS software version 13.0. Differences were considered statistically significant at $p<0.05$.

\section{Results}

Levels of miR-133b, EGFR, ITGB4, and FAK in ESCC tissue samples

At first, the miR-133b level, and EGFR, ITGB4 mRNA levels in 30 pairs of ESCC tissues and adjacent normal tissues were assessed by RT-qPCR. The clinicopathological characteristics of patients with ESCC are listed in Table 1. As illustrated in Fig. 1a, compared with in normal tissues, the level of miR-133b was decreased, while EGFR and ITGB4 mRNA levels were enhanced in tumor tissues. Moreover, western blotting analysis was performed to further verify the above results. As shown in Fig. 1b, c, the protein levels of EGFR, ITGB4 and p-FAK were increased in tumor tissues, as compared with in normal tissues. From these results, we found that miR133b level was negatively correlated with EGFR and ITGB4 levels in tumor tissues, which may participate in the metastasis of ESCC.

Levels of miR-133b, EGFR, ITGB4, and FAK in ESCC cell lines Next, we investigated the levels of miR-133b, EGFR, and ITGB4 at the cellular level in three ESCC cell lines, including KYSE150, KYSE30, and ECa109, and normal human esophageal epithelial cell line Het-1A. Consistent

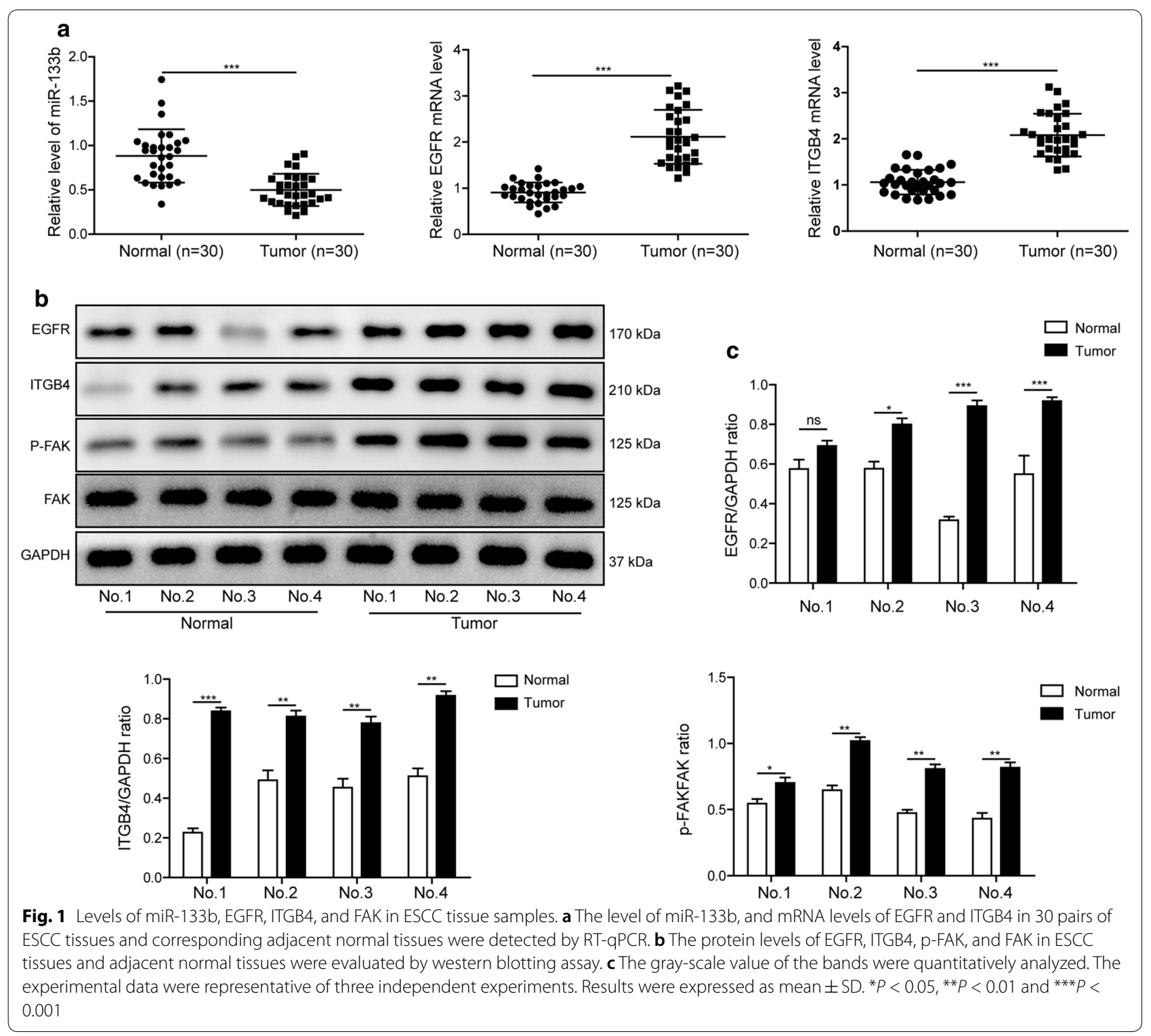


with the results in tissue samples, the level of miR-133b was reduced significantly, while EGFR and ITGB4 mRNA levels were strikingly increased in three ESCC cell lines, compared with in normal Het-1A cells (Fig. 2a). As expected, the protein levels of EGFR, ITGB4, and p-FAK in KYSE150, KYSE30, and ECa109 cells were distinctly enhanced (Fig. 2b, c). Thus, the negative relationship between miR-133b and EGFR, ITGB4 was further confirmed in ESCC cells. KYSE150 and ECa109 cells were selected for the following experiments.

\section{MiR-133b regulated EGFR expression in ESCC cell lines}

To evaluate whether EGFR is the target gene of miR$133 \mathrm{~b}$, dual luciferase reporter assay was performed. The WT or MUT 3'-UTR of EGFR was constructed to a luciferase system. The results showed that the luciferase activity of the WT $3^{\prime}$-UTR of EGFR was restrained by the overexpression of miR-133b, but that of the MUT 3'-UTR of EGFR was not affected (Fig. 2d, e). Furthermore, we investigated the regulatory role of miR-133b on EGFR expression in ESCC cells. In order to do that, agomir or antagomir of miR-133b was adopted to regulate miR-133b level and shEGFR to silence the expression of EGFR in ESCC cells. As presented in Fig. 3a, b, transfection with miR-133b agomir remarkably up-regulated cellular miR-133b level and simultaneously inhibited EGFR mRNA level in KYSE150 and ECa109 cells. Treatment with shEGFR effectively suppressed EGFR mRNA level, but had no effect on miR-133b level. However, co-transfection with shEGFR and miR-133b antagomir significantly repressed miR-133b level and reversed EGFR mRNA level induced by shEGFR (Fig. 3a, b). In addition, treatment with miR-133b agomir or shEGFR could reduce the protein level of EGFR in KYSE150 and ECa109
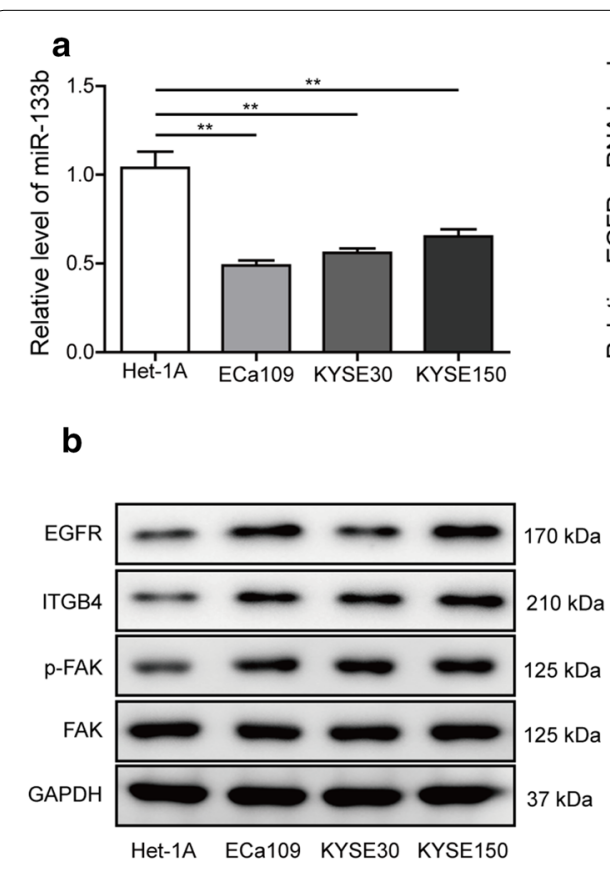

d

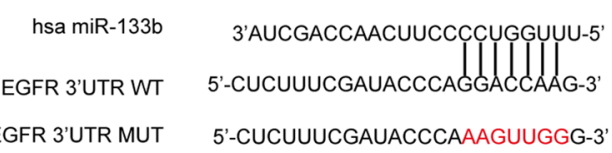

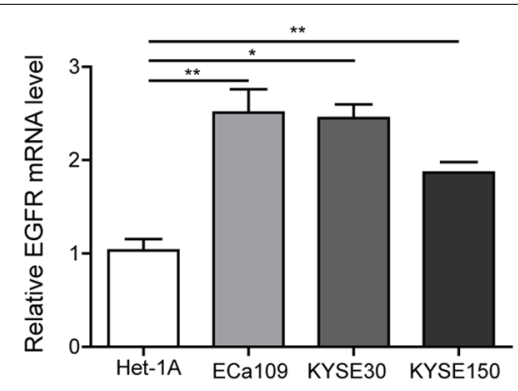
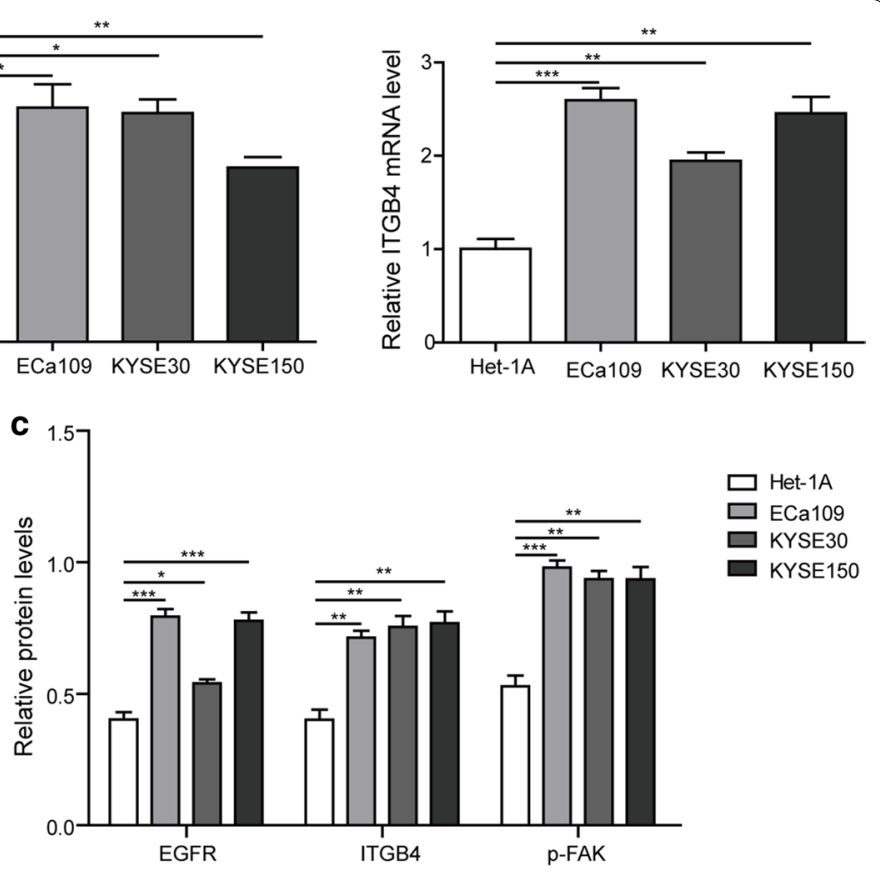

e

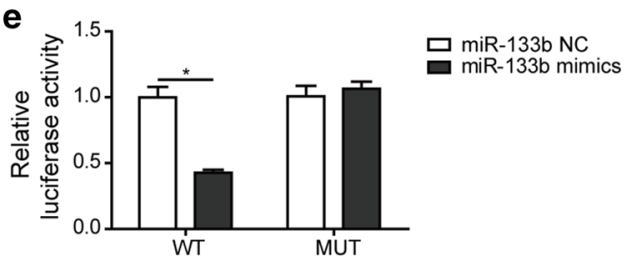

Fig. 2 Levels of miR-133b, EGFR, ITGB4, and FAK in ESCC cell lines. a The level of miR-133b, and mRNA levels of EGFR and ITGB4 in ESCC cell lines and normal human esophageal epithelial cell line were detected by RT-qPCR. $\mathbf{b}$ The protein levels of EGFR, ITGB4, p-FAK, and FAK in ESCC cell lines and normal human esophageal epithelial cell line were detected by western blotting. $\mathbf{c}$ The gray-scale value of the bands were quantitatively analyzed. $\mathbf{d}$ The specific binding sites of miR-133b in 3'-UTR of EGFR mRNA, and mutation binding sites were shown. WT, wild-type; MUT, mutant type. e Relative luciferase activities after co-transfection with the EGFR reporter and the miR-133b agomir or miR-133b NC for $48 \mathrm{~h}$. The experimental data were representative of three independent experiments. Results were expressed as mean \pm SD. ${ }^{*} P<0.05,{ }^{* *} P<0.01$ and ${ }^{* * *} P<0.001$ 


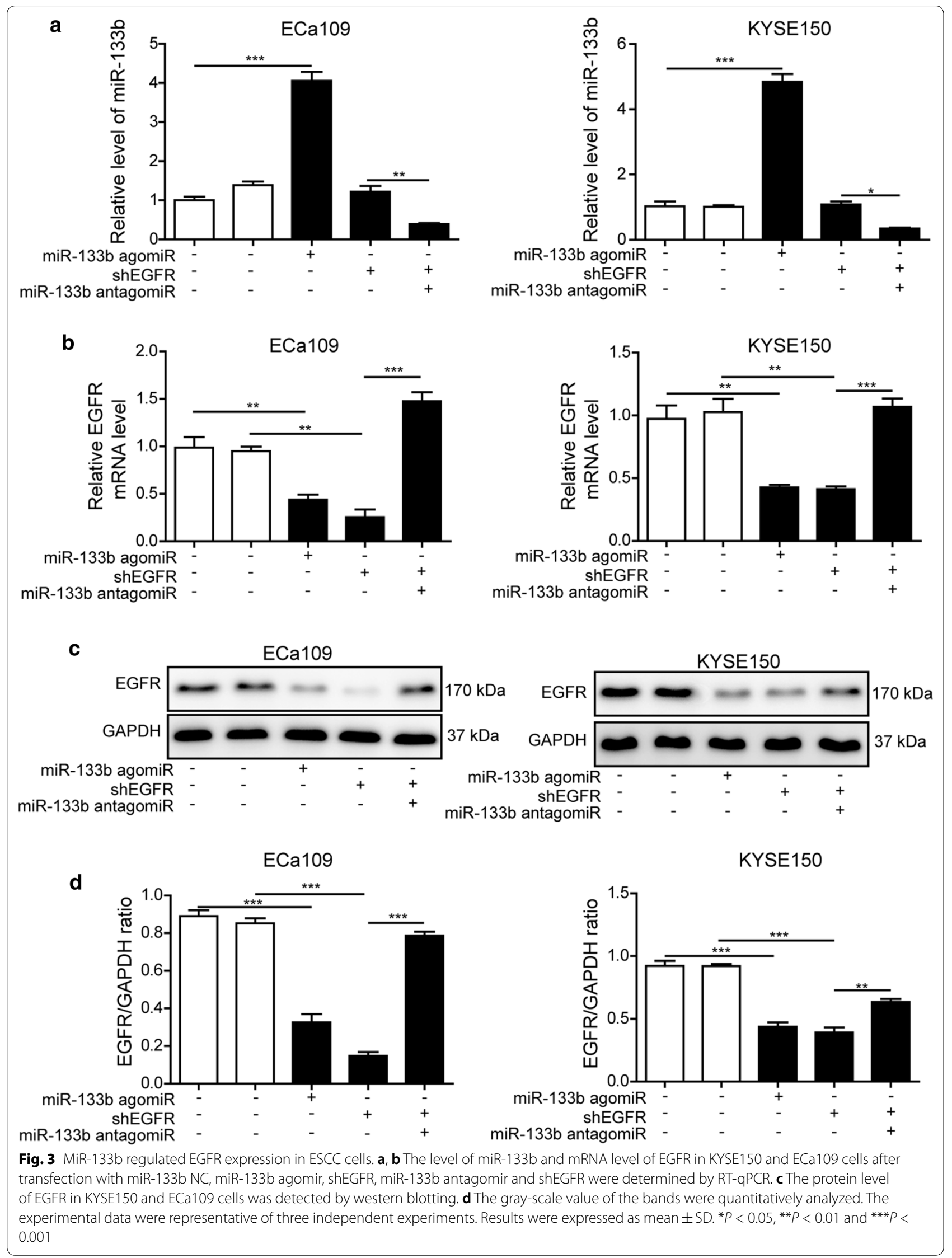


cells. Compared with in shEGFR group, the EGFR protein level was raised in shEGFR and miR-133b antagomir co-transfected group (Fig. 3c, d). According to these results, EGFR was verified to be the target gene of miR-133b in ESCC cells.

\section{MiR-133b repressed proliferation, anoikis resistance and anchorage-independent growth of ESCC cells via regulating EGFR}

The proliferation of KYSE150 and ECa109 cells was assessed by MTT assay. It was observed that cell proliferation was restrained by miR-133b agomir or shEGFR. However, miR-133b antagomir obviously inhibited the decrease in the proliferation of KYSE150 and ECa109 cells induced by shEGFR (Fig. 4a). Moreover, miR$133 \mathrm{~b}$ agomir or shEGFR treatment promoted apoptosis and repressed anoikis resistance of KYSE150 and ECa109 cells, whereas miR-133b antagomir weakened the effects of shEGFR treatment on anoikis resistance (Fig. 4b, c). As illustrated in Fig. 4d, e, cellular anchorage-independent growth was determined by soft agar cloning assay. The results indicated that KYSE150 and ECa109 cells that were transfected with miR-133b agomir or shEGFR formed much fewer colonies, compared with the cells in miR-133b NC or shNC group. However, miR-133b antagomir significantly increased the number of colonies that were formed by KYSE150 and ECa109 cells transfected with shEGFR. Thus, miR-133b significantly inhibited the proliferation, anoikis resistance and anchorage-independent growth of ESCC cells via regulating EGFR.

\section{MiR-133b suppressed migration, invasion and EMT process} of ESCC cells via regulating EGFR

The migration ability of ESCC cells was assessed by scratch assay. As presented in Fig. 5a, b, the migration ability of KYSE150 and ECa109 cells that were transfected with miR-133b agomir or shEGFR was suppressed. MiR-133b antagomir could enhance the migration ability of cells induced by shEGFR. Besides, miR-133b agomir or shEGFR evidently lessened the number of invasive KYSE150 and ECa109 cells as detected by transwell assay. But the number of invasive cells was significantly raised by miR-133b antagomir, compared with in shEGFR group (Fig. 5c, d). Moreover, the protein levels of EMT and metastasis-related markers were evaluated by western blotting analysis. As shown in Fig. 5e, the protein levels of Fibronectin, Vimentin, N-cadherin, MMP-2, and MMP-9 were decreased, while the protein level of E-cadherin was increased in KYSE150 and ECa109 cells that were treated with miR-133b agomir or shEGFR. As we expected, miR-133b antagomir could reverse shEGFR-induced the changes in these protein levels. Therefore, the migration, invasion and EMT process of ESCC cells were restrained by miR-133b via regulating EGFR.

\section{Regulation of miR-133b/EGFR axis in related signaling pathways of ESCC cells}

To elucidate the detailed mechanisms underlying the roles of miR-133b/EGFR axis in ESCC cells, a series of related molecules and signaling pathways were evaluated. As presented in Fig. $6 \mathrm{a}-\mathrm{d}$, miR-133b agomir or shEGFR caused obvious decrease in the protein levels of ITGB4,

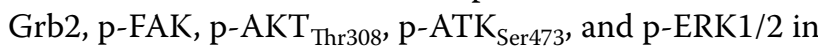
KYSE150 and ECa109 cells. But the reverse could occur, when the ESCC cells were co-transfected with miR-133b antagomir and shEGFR. Thus, ITGB4/FAK/Grb2, AKT and ERK pathways were involved in the mechanisms of miR-133b/EGFR axis in ESCC cells. MiR-133b/EGFR axis might regulate the proliferation, anoikis resistance and anchorage-independent growth, migration and invasion of ESCC cells through ITGB4/FAK/Grb2, AKT and ERK signaling pathways.

\section{MiR-133b inhibited tumor growth and lung metastasis of ESCC cells in mice}

Finally, we further verified our results in a nude mice model in vivo. The results suggested that the tumor volume and weight were decreased in mice that were injected with miR-133b overexpressing KYSE150 and ECa109 cells (Fig. 7a-c). The level of miR-133b was raised, while EGFR, ITGB4, and Grb2 mRNA levels were reduced in the lung tissues of mice that were injected with miR-133b agomir-transfected KYSE150 and ECa109 cells (Fig. 7d, e). Concurrently, miR-133b agomir treatment led to the decreased protein levels of EGFR, ITGB4, p-FAK, and Grb2 in lung tissues of mice (Fig. 7f, g). The above results suggested that miR-133b inhibited the tumor growth and lung metastasis of ESCC cells in nude mice in vivo.

\section{Discussion}

In this study, we aimed to evaluate the roles of miR-133b/ EGFR axis in the metastasis of ESCC. Our results indicated that miR-133b level was down-regulated in the tissues and cells of ESCC, which promoted the proliferation, anoikis resistance, anchorage-independent growth and eventually led to ESCC invasion and metastasis via targeting EGFR and the downstream ITGB4/FAK/Grb2, AKT and ERK pathways. These results elucidated the mechanisms of invasion and metastasis of ESCC, which was a great help to bring more new strategies for ESCC therapy at the molecular level.

It was confirmed that miR-133b was down-regulated in multiple types of cancers, including ESCC, and played 


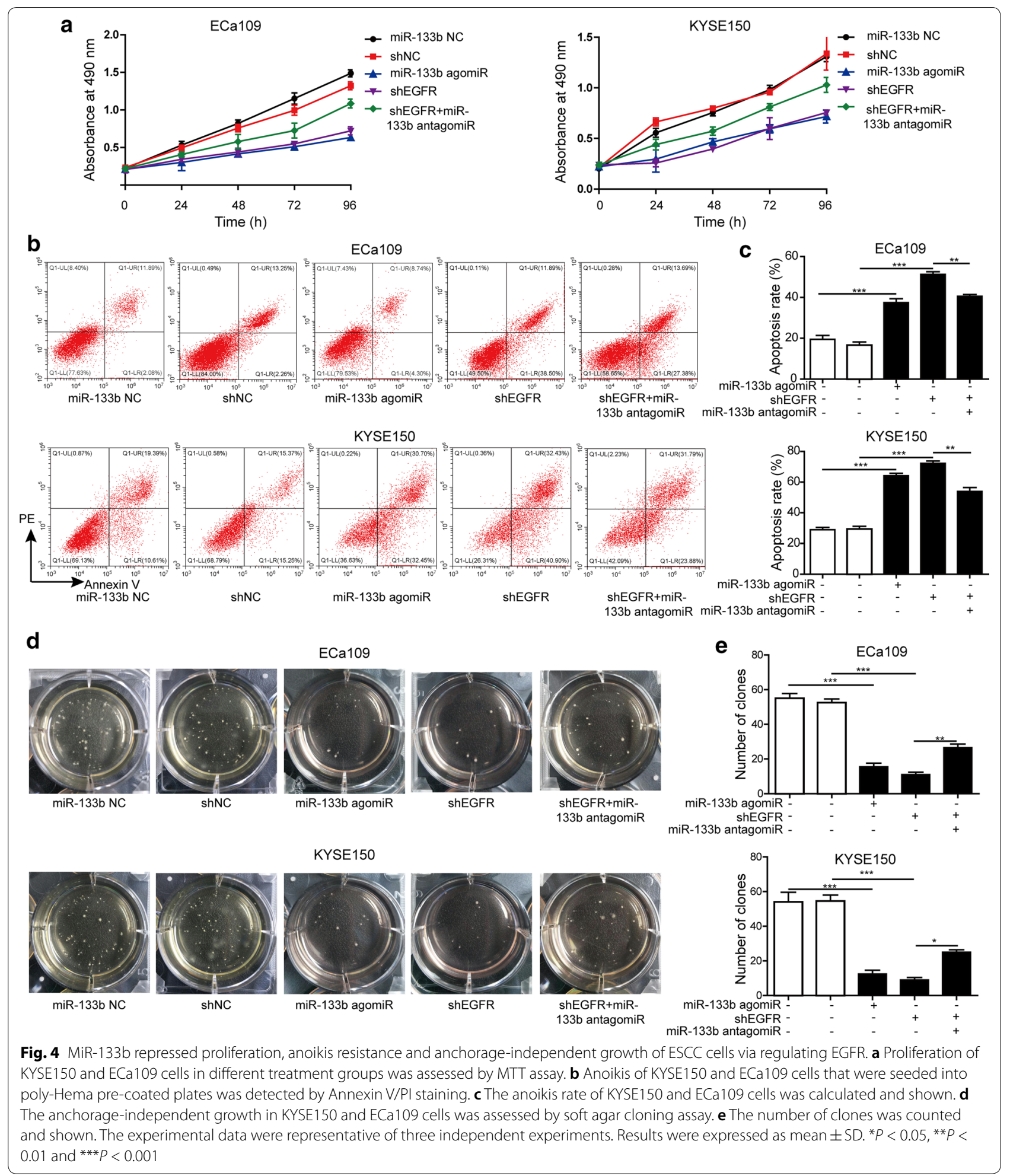

crucial roles in malignant progression of tumors. However, the detailed mechanisms of miR-133b in the regulation of invasion and metastasis of ESCC are not fully understood. Consistent with previous studies, our results indicated that the expression of miR-133b in ESCC tissues and cells was remarkably decreased. Moreover, miR133b expression was negatively correlated with EGFR, ITGB4 and p-FAK levels in the tissues and cells of ESCC, 


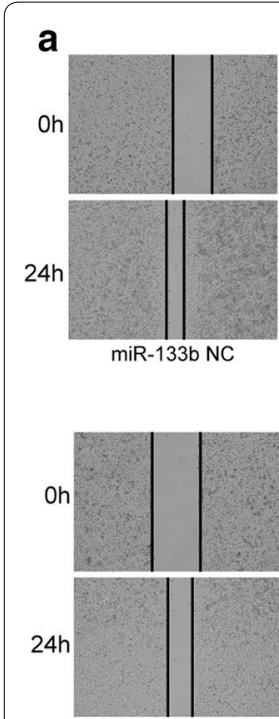

miR-133b NC
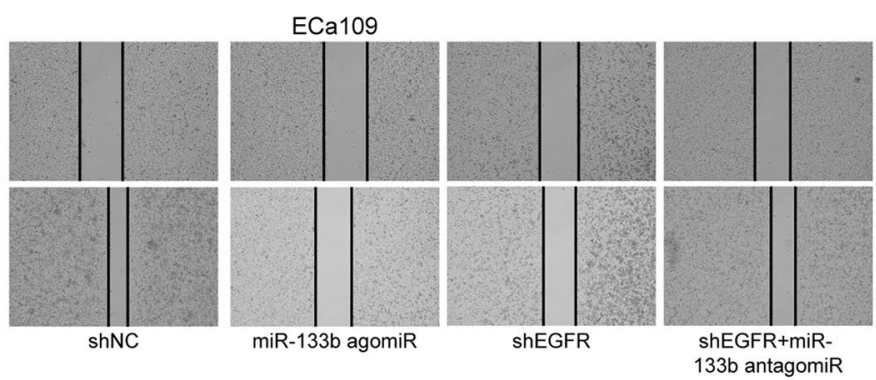

b

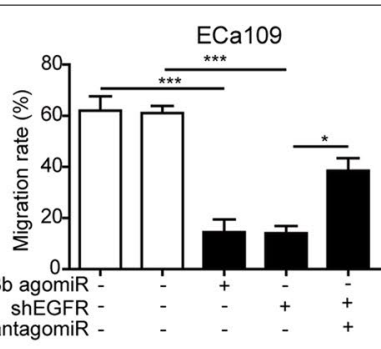

KYSE150

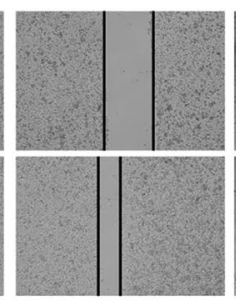

shNC
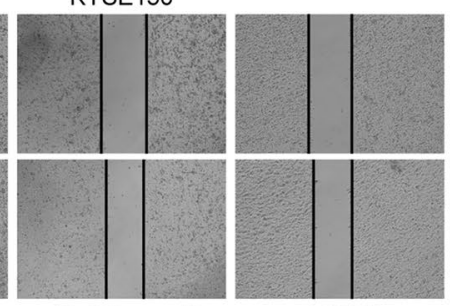

shEGFR

C

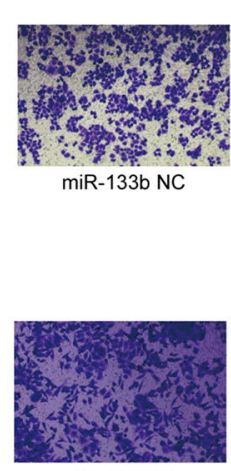

miR-133b NC

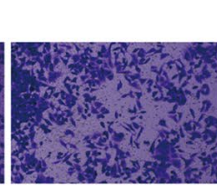

shNC

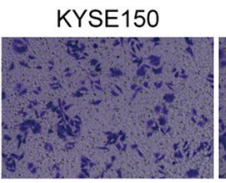

miR-133b agomiR

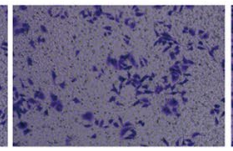

shEGFR
ECa109

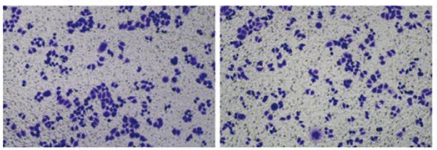

e

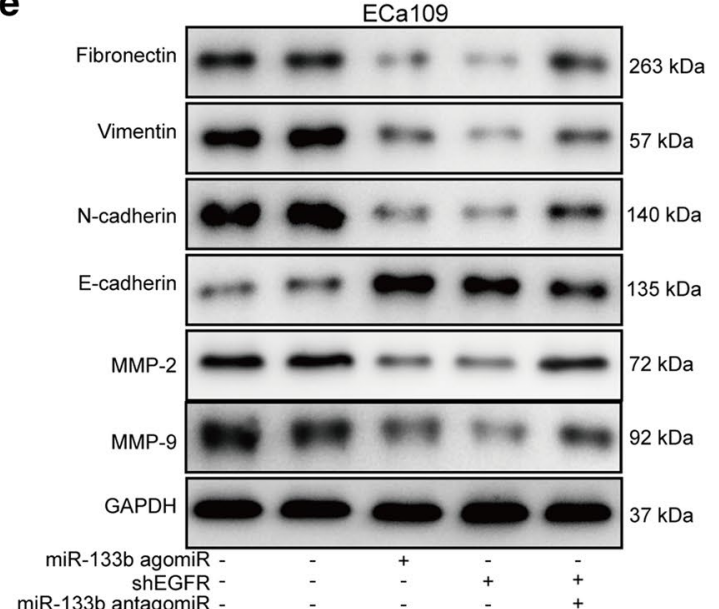

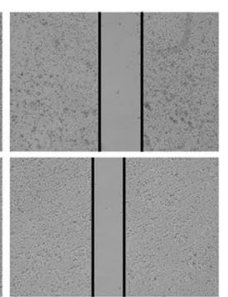

shEGFR+miR-

$133 \mathrm{~b}$ antagomiR

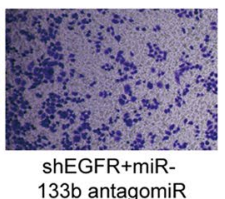

miR-133b agomiR ! ShEGFR -
miR-133b antagomiR -
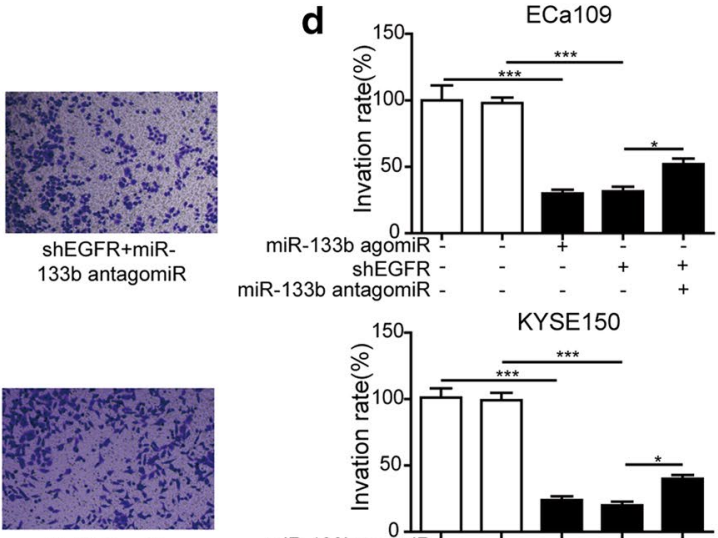

shEGFR+miR$133 \mathrm{~b}$ antagomiR

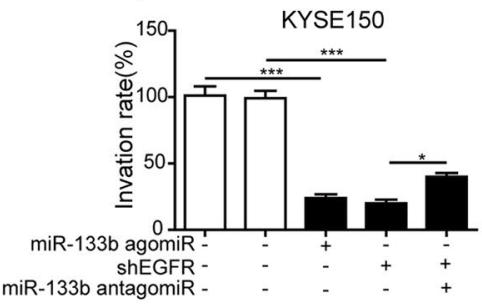

miR-133b shEGFR -
KYSE150

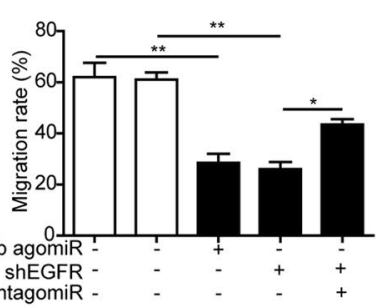
shEGFR
miR-133b antagomiR

KYSE150

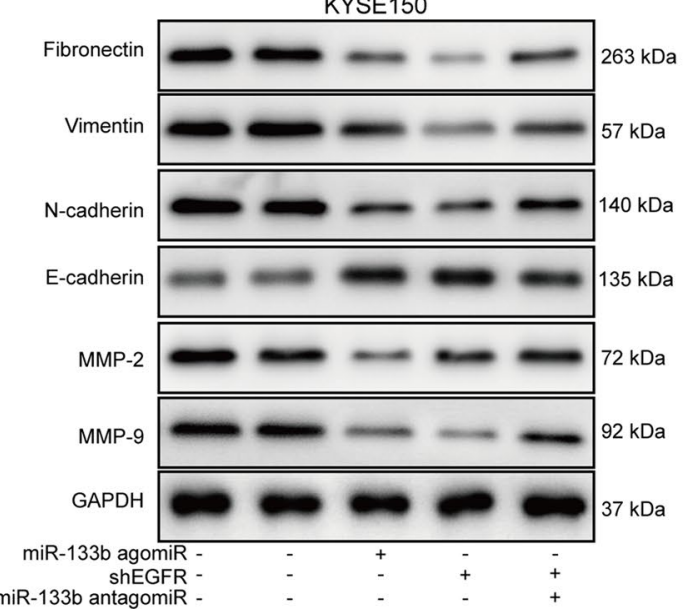

Fig. 5 MiR-133b suppressed migration, invasion and EMT process of ESCC cells via regulating EGFR. a The migration ability of KYSE150 and ECa109 cells was evaluated by scratch assay. b The migration rate was calculated and shown. c The invasion ability of KYSE150 and ECa109 cells was detected by transwell assay. $\mathbf{d}$ The number of invasive cells was counted. e The protein levels of Fibronectin, Vimentin, N-cadherin, E-cadherin, MMP-2, and MMP-9 in KYSE150 and ECa109 cells were assessed by western blotting. The experimental data were representative of three independent experiments. Results were expressed as mean $\pm S \mathrm{SD}$. ${ }^{*} P<0.05$, ${ }^{* *} P<0.01$ and ${ }^{* * *} P<0.001$ 


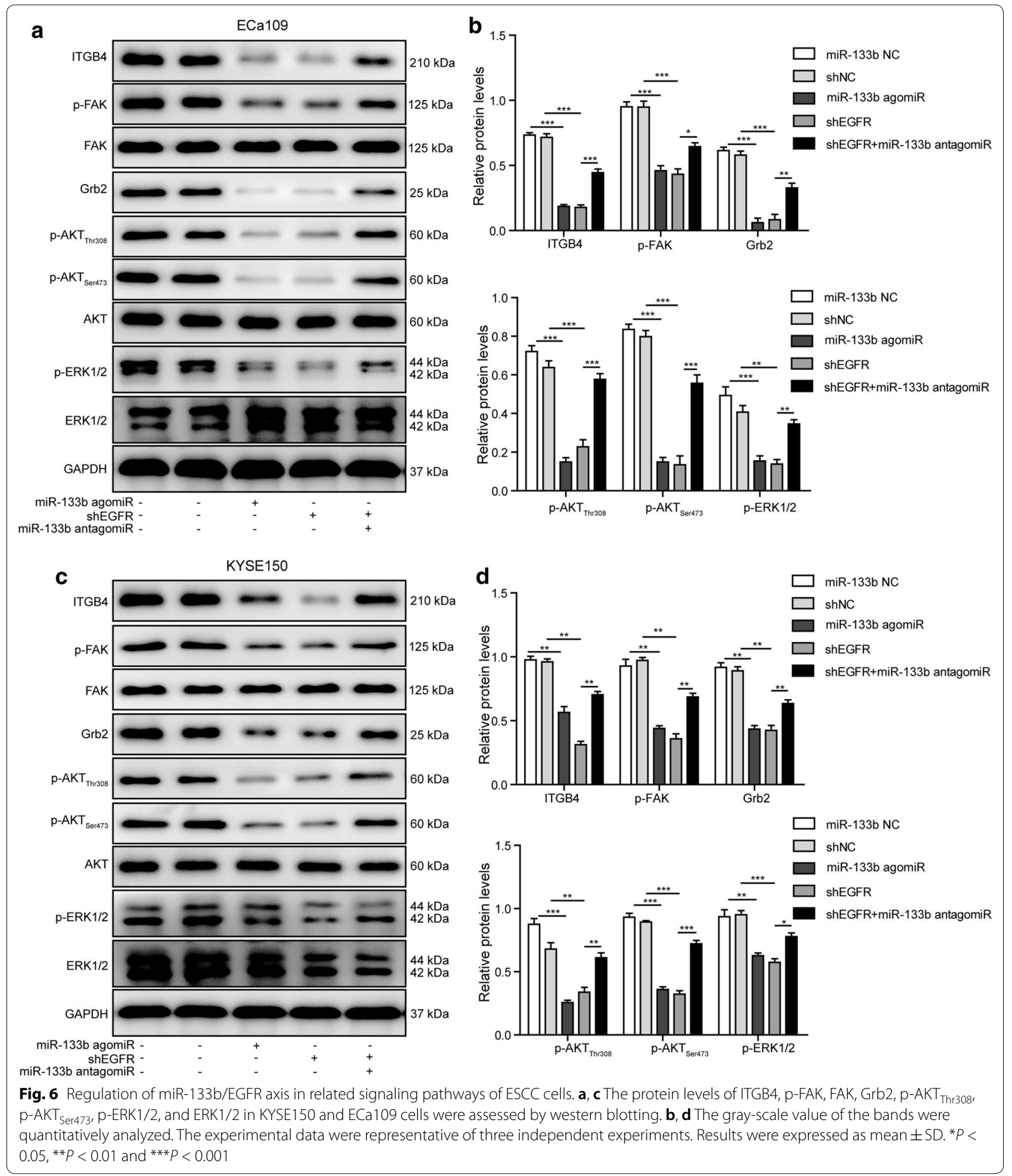

suggesting these molecules may participate in the regulatory mechanisms of miR-133b in ESCC.

EGFR has been proved to be one of target genes of miR$133 \mathrm{~b}$ in several human cancer cells $[12,13,31]$. It is well acknowledged that EGFR is a transmembrane glycoprotein with intracellular tyrosine kinase activity [32]. The activation of EGFR can trigger a series of downstream signaling pathways, principally the mitogen-activated protein kinase 


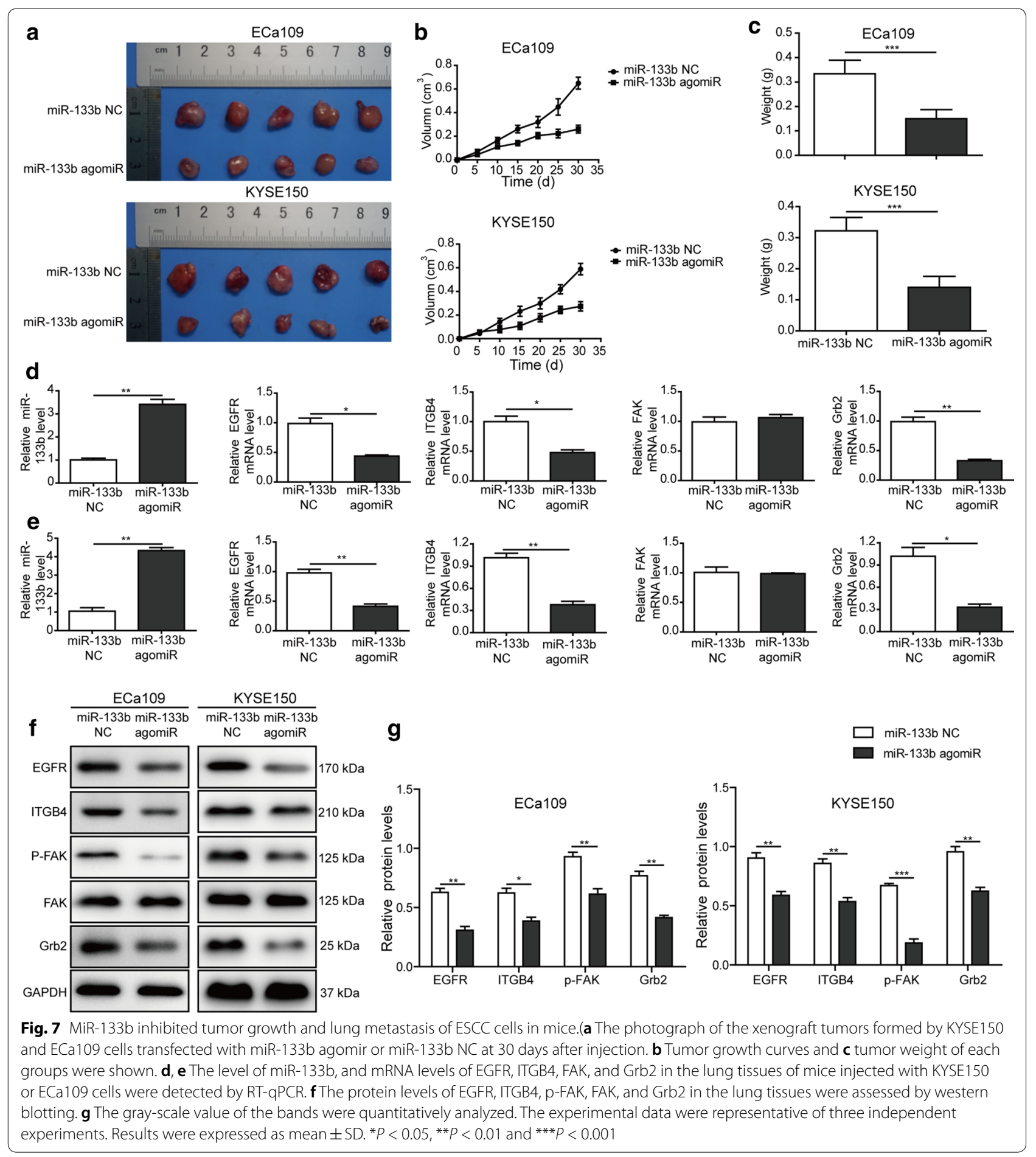

(MAPK) and PI3 K/AKT pathways [33] that are associated with proliferation, invasion and metastasis of cancer cells. Therefore, a number of anticancer drugs called "EGFR inhibitors" that targeting EGFR such as cetuximab and panitumumab have been adopted. In the present study, the results of dual luciferase reporter assay showed that EGFR was a target gene of miR-133b in ESCC cells, which further verified by the negative relationship between miR-133b and EGFR expression in ESCC tissues and cells. Moreover, overexpression of miR-133b restrained EGFR level in ESCC cells, whereas silencing of EGFR had no effect on miR-133b expression. These results provided evidence that 
in addition to EGFR, miR-133b might regulate the expression of other genes whose products were involved in EGFR signaling pathways.

Cancer cells detachment from ECM and the subsequent proliferation under anchorage-independent growth condition are considered as an early step of cancer metastasis [34]. Under normal condition, the detached normal cells may suffer apoptosis regulated by anoikis signaling pathways [35]. However, the tumor cells may survive and develop distant metastasis through anoikis resistance [36]. In this study, miR-133b overexpression and EGFR down-regulation suppressed anoikis resistance and anchorage-independent growth in ESCC cells via inhibiting EGFR expression. From these results, we speculated that miR-133b/EGFR might affect the migration and invasion of ESCC cells via regulating anoikis resistance and anchorage-independent growth. As expected, our results confirmed that the migration and invasion abilities of ESCC cells were repressed by miR-133b agomir and shEGFR via targeting EGFR. MMP-2 and MMP-9 are two important ECM degradation enzymes that promote tumor invasion and metastasis via breaking basement membrane structure and degrading ECM [37]. In this study, the protein levels of MMP-2 and MMP-9 in ESCC cells were suppressed by miR-133b overexpression and EGFR silencing. It is recognized that anoikis resistance facilitates metastasis via triggering EMT [18]. EMT is a process that well differentiated epithelial cells lose their polarity and cell-cell tight junctions, and transform to mesenchymal cells with increased motility and invasion abilities. Emerging data demonstrate that EMT is the driving force of cancer metastasis [38, 39]. During EMT process, the expression of E-cadherin, a epithelial marker, is decreased, while the expressions of mesenchymal markers, Fibronectin, Vimentin, and $\mathrm{N}$-cadherin, are demonstrated to be increased [40]. According to the present study, the protein levels of Fibronection, Vimentin, and $\mathrm{N}$-cadherin were down-regulated, while E-cadherin level was up-regulated in ESCC cells by miR-133b agomir or shEGFR treatment via targeting modulation of EGFR. Also, miR-133b antagomir reversed shEGFRinduced the changes in proliferation, anoikis resistance and anchorage-independent growth, migration and invasion of ESCC cells. From these results, we proposed that miR-133b/EGFR axis played pivotal roles in metastasis of ESCC via regulating anoikis resistance and anchorageindependent growth.

Furthermore, we focused on the detailed molecular mechanisms of miR-133b/EGFR axis in the regulation of anoikis resistance and metastasis of ESCC. Since we demonstrated that miR-133b expression was negatively correlated with ITGB4 and p-FAK levels in ESCC tissues and cells, the effects of miR-133b on ITGB4, FAK and related signaling pathways were further investigated. ITGB4 has been suggested to be up-regulated in multiple tumors and contribute to tumor progression by promoting proliferation, invasion and EMT process [41, 42]. A previous study showed that ITGB4 affected anoikis through interacting with EGFR in hepatocellular carcinoma [25]. ITGB4 was found to trigger the activation of downstream transmembrane protein kinases, including FAK, to protect against anoikis [43]. In addition, it was confirmed that FAK could bind to Grb2 and regulate the proliferation and invasion of melanoma [28]. There were a lot of downstream signaling pathways, such as AKT and ERK, that were regulated by Grb2 and facilitated tumor progression [30, 38]. In our study, miR-133b agomir or shEGFR inhibited the levels of ITGB4, Grb2, and the phosphorylation level of FAK, AKT and ERK. Our results indicated that miR-133b/ EGFR axis regulated ITGB4/FAK/Grb2 pathway and downstream AKT and ERK pathways in ESCC cells, which might involve in the mechanisms of anoikis resistance and metastasis.

To further verify our findings, tumor xenografts in nude mice were performed. Consistent with the in vitro results, overexpression of miR-133b significantly restrained the tumor growth and lung metastases via regulating EGFR/ITGB4/FAK/Grb2 signaling pathway.

\section{Conclusions}

Taken together, our data suggested that miR-133b was down-regulated in the tissues and cells of ESCC, which negatively correlated with EGFR, ITGB4, and p-FAK levels. Moreover, miR-133b inhibited the metastases of ESCC by regulating anoikis and anchorage-independent growth via targeting EGFR in vitro and in vivo. ITGB4/FAK/Grb2 and downstream AKT and ERK pathways participated in the regulatory mechanisms of miR-133b/EGFR axis in ESCC. Collectively, our results shed lights on the roles and mechanisms of miR-133b in ESCC metastases, which were involved in modulation of anoikis resistance and anchorage-independent growth. MiR-133b may be a potential novel diagnostic and therapeutic target against this malignancy.

\section{Abbreviations}

ESCC: esophageal squamous cell carcinoma; miRNAs: microRNAs; UTRs: untranslated regions; EGFR: epidermal growth factor receptor; ECM: extracellular matrix; ITGB4: integrin $\beta 4$; FAK: focal adhesion kinase; PI3K: phosphatidylinositol 3-kinase; AKT: protein kinase B; Grb2: growth factor receptor-bound protein 2; ERK: extracellular signal-regulated kinase; RPMI: Roswell Park

Memorial Institute; FBS: fetal bovine serum; DMEM: Dulbecco's Modified Eagle Medium; WT: wild-type; MUT: mutated; MTT: 3-(4,5-dimethylthiazol-2-yl)-

2,5-diphenyltetrazolium bromide; DMSO: dimethyl sulfoxide; PBS: phosphate buffer solution; RT-qPCR: reverse transcription-quantitative polymerase chain reaction; GAPDH: glyceraldehyde-3-phosphate dehydrogenase; snRNA: small nuclear RNA; RIPA: radio immunoprecipitation assay; PMSF: phenylmethanesulfonyl fluoride; BCA: bicinchoninic acid; SDS-PAGE: sodium dodecyl sulphate-polyacrylamide gel electrophoresis; MMP-2: matrix metalloproteinase 2; 
SD: standard deviation; ANOVA: analysis of variance; EMT: epithelial-mesenchymal transition; MAPK: mitogen-activated protein kinase.

\section{Authors' contributions}

ZJF designed the study, prepared, edited and reviewed the manuscript. LY, HZG, LJY, DX and LYL performed experimental studies. HH and SL did literature research and data analysis. ZW designed the study and reviewed the manuscript. All authors read and approved the final manuscript.

\section{Author details \\ ${ }^{1}$ First Department of Lung Cancer Chemotherapy, Affiliated Cancer Hospital of Xinjiang Medical University, No. 789, East Suzhou Street, Urumqi 830011, Xinjiang, People's Republic of China. ${ }^{2}$ Department of Gastrointestinal Surgery, Affiliated Cancer Hospital of Xinjiang Medical University, Urumqi 830011 , People's Republic of China. ${ }^{3}$ Department of Cardiothoracic Surgery, Shenzhen University General Hospital, Shenzhen 518055, People's Republic of China. ${ }^{4}$ Department of Histology and Embryology, Xiangya School of Medicine, Central South University, Changsha 410013, People's Republic of China. ${ }^{5}$ Department of Histology and Embryology, Xinjiang Medical University, Urumqi 830011, People's Republic of China. ${ }^{6}$ Institute of Cancer Prevention and Treatment, Affiliated Cancer Hospital of Xinjiang Medical University, Urumqi 830011, People's Republic of China. ${ }^{7}$ Department of Hematology and Oncology, Shenzhen University General Hospital, No.1098, Xueyuan Avenue, Shenzhen 518055, Guangdong, People's Republic of China.}

\section{Acknowledgements}

We would like to give our sincere gratitude to the reviewers for their constructive comments.

\section{Competing interests}

The authors declare that they have no competing interests.

\section{Availability of data and materials}

All data generated or analysed during this study are included in this published article.

\section{Consent for publication}

Not applicable.

\section{Ethics approval and consent to participate}

Not applicable.

\section{Funding}

This study was supported by a grant from National Natural Science Foundation of China (No. 81660397).

\section{Publisher's Note}

Springer Nature remains neutral with regard to jurisdictional claims in published maps and institutional affiliations.

Received: 27 August 2018 Accepted: 9 November 2018 Published online: 22 November 2018

\section{References}

1. Jemal A, Bray F, Center MM, Ferlay J, Ward E, Forman D. Global cancer statistics. CA Cancer J Clin. 2011;61(2):69-90.

2. Lin Y, Totsuka Y, He Y, Kikuchi S, Qiao Y, Ueda J, Wei W, Inoue M, Tanaka $\mathrm{H}$. Epidemiology of esophageal cancer in Japan and China. J Epidemiol. 2013;23(4):233-42.

3. Engel LS, Chow WH, Vaughan TL, Gammon MD, Risch HA, Stanford JL, et al. Population attributable risks of esophageal and gastric cancers. J Natl Cancer Inst. 2003;95(18):1404-13.

4. Pennathur A, Gibson MK, Jobe BA, Luketich JD. Oesophageal carcinoma. Lancet. 2013;381(9864):400-12.

5. Rustgi AK, El-Serag HB. Esophageal carcinoma. N Engl J Med. 2014;371(26):2499-509.

6. Yang L, Hou J, Cui XH, Suo LN, Lv YW. MiR-133b regulates the expression of CTGF in epithelial-mesenchymal transition of ovarian cancer. Eur Rev Med Pharmacol Sci. 2017;21(24):5602-9.
7. Wang X, Bu J, Liu X, Wang W, Mai W, Lv B, Zou J, Mo X, Li X, Wang J, et al. miR-133b suppresses metastasis by targeting HOXA9 in human colorectal cancer. Oncotarget. 2017;8(38):63935-48.

8. Cheng Y, Jia B, Wang Y, Wan S. miR-133b acts as a tumor suppressor and negatively regulates ATP citrate lyase via PPARgamma in gastric cancer. Oncol Rep. 2017;38(5):3220-6.

9. Fu HL, Wu DP, Wang XF, Wang JG, Jiao F, Song LL, Xie H, Wen XY, Shan HS, Du YX, et al. Altered miRNA expression is associated with differentiation, invasion, and metastasis of esophageal squamous cell carcinoma (ESCC) in patients from Huaian, China. Cell Biochem Biophys. 2013;67(2):657-68.

10. Huang H, Xu Y, Guo Z, Chen X, Ji S, Xu Z. MicroRNA-133b inhibits cell proliferation and promotes apoptosis by targeting cullin 4B in esophageal squamous cell carcinoma. Exp Ther Med. 2018;15(4):3743-50.

11. Liu Q, Yu S, Zhao W, Qin S, Chu Q, Wu K. EGFR-TKIs resistance via EGFRindependent signaling pathways. Mol Cancer. 2018;17(1):53.

12. Liu L, Shao X, Gao W, Zhang Z, Liu P, Wang R, Huang P, Yin Y, Shu Y. MicroRNA-133b inhibits the growth of non-small-cell lung cancer by targeting the epidermal growth factor receptor. FEBS J. 2012;279(20):3800-12.

13. Zhou J, Lv L, Lin C, Hu G, Guo Y, Wu M, Tian B, Li X. Combinational treatment with microRNA133b and cetuximab has increased inhibitory effects on the growth and invasion of colorectal cancer cells by regulating EGFR. Mol Med Rep. 2015;12(4):5407-14.

14. Liu X, Li G. MicroRNA-133b inhibits proliferation and invasion of ovarian cancer cells through Akt and Erk1/2 inactivation by targeting epidermal growth factor receptor. Int J Clin Exp Pathol. 2015;8(9):10605-14.

15. Frisch SM, Francis H. Disruption of epithelial cell-matrix interactions induces apoptosis. J Cell Biol. 1994;124(4):619-26.

16. Grossmann J. Molecular mechanisms of "detachment-induced apoptosis-Anoikis". Apoptosis. 2002;7(3):247-60.

17. Haemmerle M, Taylor ML, Gutschner T, Pradeep S, Cho MS, Sheng J, Lyons YM, Nagaraja AS, Dood RL, Wen Y, et al. Platelets reduce anoikis and promote metastasis by activating YAP1 signaling. Nat Commun. 2017:8(1):310.

18. Paoli P, Giannoni E, Chiarugi P. Anoikis molecular pathways and its role in cancer progression. Biochem Biophys Acta. 2013;1833(12):3481-98.

19. Song KJ, Jeon SK, Moon SB, Park JS, Kim JS, Kim J, Kim S, An HJ, Ko JH, Kim YS. Lectin from Sambucus sieboldiana abrogates the anoikis resistance of colon cancer cells conferred by $\mathrm{N}$-acetylglucosaminyltransferase $\mathrm{V}$ during hematogenous metastasis. Oncotarget. 2017;8(26):42238-51.

20. Hu B, Zhang T, An HM, Zheng JL, Yan X, Huang XW. Herbal formula YGJDSJ inhibits anchorage-independent growth and induces anoikis in hepatocellular carcinoma Bel-7402 cells. BMC Complement Altern Med. 2018;18(1):17

21. Guadamillas MC, Cerezo A, Del Pozo MA. Overcoming anoikis - pathways to anchorage-independent growth in cancer. J Cell Sci. 2011;124(Pt 19):3189-97.

22. Zhang P, Song Y, Sun Y, Li X, Chen L, Yang L, Xing Y. AMPK/GSK3beta/betacatenin cascade-triggered overexpression of CEMIP promotes migration and invasion in anoikis-resistant prostate cancer cells by enhancing metabolic reprogramming. FASEB J. 2018;32(7):3924-35.

23. Salvi A, Thanabalu T. WIP promotes in vitro invasion ability, anchorage independent growth and EMT progression of A549 lung adenocarcinoma cells by regulating RhoA levels. Biochem Biophys Res Commun. 2017:482(4):1353-9.

24. Leng C, Zhang ZG, Chen WX, Luo HP, Song J, Dong W, Zhu XR, Chen XP, Liang HF, Zhang BX. An integrin beta4-EGFR unit promotes hepatocellular carcinoma lung metastases by enhancing anchorage independence through activation of FAK-AKT pathway. Cancer Lett. 2016;376(1):188-96.

25. Saranya J, Shilpa G, Raghu KG, Priya S. Morus alba leaf lectin (MLL) sensitizes MCF-7 Cells to anoikis by inhibiting fibronectin mediated integrinFAK signaling through ras and activation of P(38) MAPK. Front Pharmacol. 2017;8:34.

26. Giubellino A, Burke TR Jr, Bottaro DP. Grb2 signaling in cell motility and cancer. Expert Opin Ther Targets. 2008;12(8):1021-33.

27. Mahauad-Fernandez WD, Okeoma CM. Cysteine-linked dimerization of BST-2 confers anoikis resistance to breast cancer cells by negating proapoptotic activities to promote tumor cell survival and growth. Cell Death Dis. 2017;8(3):e2687.

28. Nihal M, Wood GS. C-CBL regulates melanoma proliferation, migration, invasion and the FAK-SRC-GRB2 nexus. Oncotarget. 2016;7(33):53869-80. 
29. Liang $C, X u Y$, Ge H, Xing B, Li G, Li G, Wu J. miR-564 inhibits hepatocellular carcinoma cell proliferation and invasion by targeting the GRB2-ERK1/2AKT axis. Oncotarget. 2017;8(64):107543-57.

30. Chen D, Si W, Shen J, Du C, Lou W, Bao C, Zheng H, Pan J, Zhong G, Xu $L$, et al. miR-27b-3p inhibits proliferation and potentially reverses multichemoresistance by targeting CBLB/GRB2 in breast cancer cells. Cell Death Dis. 2018;9(2):188

31. Tao J, Wu D, Xu B, Qian W, Li P, Lu Q, Yin C, Zhang W. microRNA-133 inhibits cell proliferation, migration and invasion in prostate cancer cells by targeting the epidermal growth factor receptor. Oncol Rep. 2012;27(6):1967-75

32. Huang CW, Chen YT, Tsai HL, Yeh YS, Su WC, Ma CJ, Tsai TN, Wang JY. EGFR expression in patients with stage III colorectal cancer after adjuvant chemotherapy and on cancer cell function. Oncotarget. 2017;8(70):114663-76.

33. Oda K, Matsuoka Y, Funahashi A, Kitano H. A comprehensive pathway map of epidermal growth factor receptor signaling. Mol Syst Biol. 2005;2005(1):0010.

34. Fidler IJ. The pathogenesis of cancer metastasis: the 'seed and soil' hypothesis revisited. Nat Rev Cancer. 2003;3(6):453-8.

35. Okayama H. Cell cycle control by anchorage signaling. Cell Signal. 2012;24(8):1599-609.
36. Simpson CD, Anyiwe K, Schimmer AD. Anoikis resistance and tumor metastasis. Cancer Lett. 2008;272(2):177-85.

37. Sounni NE, Noel A. Membrane type-matrix metalloproteinases and tumor progression. Biochimie. 2005;87(3-4):329-42.

38. Cao XY, Zhang XX, Yang MW, Hu LP, Jiang SH, Tian GA, Zhu LL, Li Q, Sun YW, Zhang ZG. Aberrant upregulation of KLK10 promotes metastasis via enhancement of EMT and FAK/SRC/ERK axis in PDAC. Biochem Biophys Res Commun. 2018;499(3):584-93.

39. Liu W, Wang $X$, Wang Y, Dai Y, Xie Y, Ping Y, Yin B, Yu P, Liu Z, Duan $X$, et al. SGK1 inhibition-induced autophagy impairs prostate cancer metastasis by reversing EMT. J Exp Clin Cancer Res. 2018;37(1):73.

40. Kyung SY, Kim DY, Yoon JY, Son ES, Kim YJ, Park JW, Jeong SH. Sulforaphane attenuates pulmonary fibrosis by inhibiting the epithelialmesenchymal transition. BMC Pharmacol Toxicol. 2018;19(1):13.

41. Guo W, Pylayeva Y, Pepe A, Yoshioka T, Muller WJ, Inghirami G, Giancotti FG. Beta 4 integrin amplifies ErbB2 signaling to promote mammary tumorigenesis. Cell. 2006;126(3):489-502.

42. Hsu YL, Wu CY, Hung JY, Lin YS, Huang MS, Kuo PL. Galectin-1 promotes lung cancer tumor metastasis by potentiating integrin alpha6beta4 and Notch1/Jagged2 signaling pathway. Carcinogenesis. 2013;34(6):1370-81.

43. Geiger TR, Peeper DS. Metastasis mechanisms. Biochim et Biophys Acta. 2009:1796(2):293-308.
Ready to submit your research? Choose BMC and benefit from:

- fast, convenient online submission

- thorough peer review by experienced researchers in your field

- rapid publication on acceptance

- support for research data, including large and complex data types

- gold Open Access which fosters wider collaboration and increased citations

- maximum visibility for your research: over $100 \mathrm{M}$ website views per year

At BMC, research is always in progress.

Learn more biomedcentral.com/submissions 